\title{
LA RAGE EN FRANCE : VIEUX PROBLÈME, NOUVELLE CRISE
}

\author{
Franck Foures
}

De Boeck Université | Politix

$2010 / 2-n^{\circ} 90$

pages 167 à 191

ISSN 0295-2319

ISBN 9782804161200

Article disponible en ligne à l'adresse:

http://www.cairn.info/revue-politix-2010-2-page-167.htm

Pour citer cet article :

Foures Franck, «La rage en France : vieux problème, nouvelle crise »,

Politix, 2010/2 n 90, p. 167-191. DOI : 10.3917/pox.090.0167

Distribution électronique Cairn.info pour De Boeck Université.

(c) De Boeck Université. Tous droits réservés pour tous pays.

La reproduction ou représentation de cet article, notamment par photocopie, n'est autorisée que dans les limites des conditions générales d'utilisation du site ou, le cas échéant, des conditions générales de la licence souscrite par votre établissement. Toute autre reproduction ou représentation, en tout ou partie, sous quelque forme et de quelque manière que ce soit, est interdite sauf accord préalable et écrit de l'éditeur, en dehors des cas prévus par la législation en vigueur en France. II est précisé que son stockage dans une base de données est également interdit. 


\section{VARIA}




\title{
La rage en France : vieux problème, nouvelle crise
}

\author{
Franck Foures
}

Résumé - En août 2004 éclate pour la première fois une crise liée à l'introduction en France d'un chien enragé. De multiples événements similaires sont pourtant toujours restés confinés dans la sphère professionnelle. Pour éclairer la genèse de cette première crise de la rage, cet article montre comment cette transformation d'un " vieux problème public " en crise peut être interprétée comme le résultat d'une brusque renégociation du compromis historique tissé autour de la gestion de cette maladie. Les recompositions de la scène de la sécurité sanitaire qui précèdent la crise vont permettre aux organisations de la Santé de se repositionner au cœur d'un dispositif de gestion de la rage dont elles étaient restées en marge. La succession des événements sanitaires, canicule tout d'abord et alertes à la rage ensuite, en remodelant la perception des crises et en développant des savoirs spécifiques sur la rage vont parallèlement rendre pensable et possible la transformation d'une alerte à la rage en crise. Enfin, la confrontation de cadres d'action différents va contribuer à reconfigurer le regard et l'analyse du risque sur l'alerte d'août 2004 et ainsi radicaliser les mesures de gestion mises en œuvre. Aussi comprendre la crise de la rage d'août 2004 nécessite-t-il de la replacer à la fois au sein de l'histoire du problème public de la rage et au cœur des reconfigurations inhérentes à l'ensemble des crises sanitaires. 
$\mathrm{E}$ n août 2004, la découverte à la société protectrice des animaux (SPA) de Bordeaux de Tikki, une jeune chienne enragée, devient en quelques jours un sujet d'envergure nationale. Tikki fait d'emblée et plusieurs jours de suite l'ouverture des journaux télévisés des grandes chaînes nationales ${ }^{1}$. Pendant plusieurs semaines, les nouvelles de cette maladie qui menace essentiellement l'Aquitaine sont omniprésentes sur les ondes, dans la presse tant nationale que régionale ${ }^{2}$. En l'espace de quelques jours, les autorités sanitaires multiplient les interventions et les ministres de la Santé et de l'Agriculture s'opposent par médias interposés sur les modalités de gestion de ce que tous les acteurs qualifient unanimement de "crise rage ». Alors que de nombreux cas de rage en France sont passés inaperçus, on assiste en août 2004 à la première crise relative à cette maladie en France.

Comment expliquer ce phénomène ? Doit-on, comme le suggèrent les protagonistes de la crise, chercher l'explication dans les spécificités de l'épisode d'août 2004 ? Serait-on simplement face à un cas exceptionnel justifiant en soi cette réponse exceptionnelle ? Faut-il, au contraire, voir dans ce traitement la conséquence d'un surinvestissement médiatique ou encore d'une forme d'hypersensibilité de la société aux risques sanitaires ? Pour bien saisir l'originalité de l'apparition de cette crise et les questionnements qu'elle induit, il faut avoir en tête plusieurs éléments d'information. Premièrement, la rage, contrairement à l'encéphalopathie spongiforme bovine (ESB) ou au syndrome respiratoire aigu sévère (SRAS), n'est pas une maladie nouvelle ou exotique susceptible de confronter les autorités à des questions radicalement nouvelles. La rage, maladie historique, connue pour son caractère mortel pour l'homme et les mammifères, a disparu en France en 1941 pour resurgir et s'y installer à nouveau pendant trente ans entre 1968 et 1998. La France n'a été reconnue officiellement indemne de rage qu'en 2000 : cette maladie est donc banale en France, à peine quelques années avant le cas d'août 2004. Deuxièmement, il convient de relativiser son caractère épidémique, car la rage se transmet essentiellement par morsure ou griffure (et non par voie aérienne comme nombre de virus). Ainsi, au cours des trente années de présence de la rage en France, grâce à la surveillance des animaux et au traitement des personnes mordues plusieurs milliers d'animaux enragés ont circulé sur le territoire français sans jamais occasionner de décès humain ${ }^{3}$. Une épidémie de rage humaine en France n'est donc pas à craindre. Enfin, on peut nuancer le caractère surprenant ou atypique de l'épisode Tikki. On recense ainsi sept cas de chiens importés enragés en France entre 1998 et

1. Six reportages dans les journaux télévisés nationaux français dans le mois suivant l'alerte.

2. Le comptage des références à la crise rage dans les médias (presse écrite, télévision, radio) entre le 26 août et le 15 septembre 2004 fait apparaître trois cent communications (source Factiva : cent vingt et une entrées complétées par les revues de presse du ministère de l'Agriculture et de la Préfecture de Gironde, soit cent soixante dix-neuf entrées non redondantes).

3. Au moment de la crise, le dernier cas humain de rage contractée en France remonte à 1924. 
2004. En particulier quelques mois avant le cas d'août $2004^{4}$, deux chiens enragés originaires eux aussi du Maroc sont retrouvés sur le sol français. Ces deux cas se règlent en quelques jours et ne font l'objet d'aucune couverture médiatique nationale. Alors, comment expliquer l'ampleur inédite donnée au cas d'août 2004 et ainsi rendre compte de cette étrange chaîne d'événements qui part d'une petite chienne ramenée sous le manteau et arrive dans les bureaux ministériels et au sein des rédactions des plus grands médias français?

Cette soudaine apparition d'un phénomène unanimement qualifié de crise par les protagonistes du dossier, dans la gestion d'un problème public ancien, parfaitement codifié et relativement usuel, fait de l'épisode de rage d'août 2004 un cas d'étude idéal. L'épisode Tikki permet d'étudier l'origine de ce processus de brusque accélération du traitement d'un problème public qui caractérise pour les gestionnaires du risque le passage d'une alerte à l'état de crise. Le cadrage du problème rage a eu lieu bien avant les bouleversements de la scène sanitaire, suite à l'ESB et au sang contaminé qui ont conduit à la mise en place des dispositifs liés à la loi de 1998 sur la veille sanitaire créant l'Agence française de sécurité des aliments (AFSSA) et l'Institut national de veille sanitaire $(\mathrm{InVS})^{5}$. La rage resurgit ainsi en 2004 en pleine restructuration de la scène sanitaire, scène secouée par les fortes remises en cause politiques qui suivent la canicule d'août $2003^{6}$. L'ampleur particulière donnée à l'épisode Tikki peut donc être interprétée comme la conséquence des tensions liées à la brusque renégociation du cadrage historique d'un vieux risque au contact d'une nouvelle scène technique et politique.

Pour mettre au jour et analyser ces tensions, ce travail s'appuie sur les tableaux de bord qui retracent la chronologie de la crise et les dossiers administratifs du ministère de l'Agriculture relatifs à l'ensemble des épisodes rage récents ${ }^{7}$. Il est complété par un corpus de quarante et un entretiens. Cet article, après une présentation du cas Tikki, retrace la problématisation historique du risque rage entre 1968 et 1998 et permet ainsi de cerner comment la politique de lutte contre la rage s'est imposée en France comme une politique d'envergure nationale pilotée par le ministère de l'Agriculture avant de s'étioler progressivement. Ce préalable permet ensuite de décrire les évolutions organisationnelles et cognitives survenues entre 1998 et 2004 offrant aux instances de la Santé de se repositionner dans la gestion du dossier rage. Enfin, l'étude spécifique du cas

\footnotetext{
4. Respectivement en janvier, puis en mai 2004.

5. Loi du $1^{\text {er }}$ juillet 1998 relative au renforcement de la veille sanitaire et au contrôle de la sécurité sanitaire des produits destinés à l'homme.

6. Lagadec (P.), Laroche (H.), Retour sur les rapports d'enquête et d'expertise suite à la canicule de l'été 2003, Grenoble, Publications de la MSH-Alpes, 2005 ; Milet (M.), "Cadres de perception et luttes d'imputation dans la gestion de la crise : l'exemple de la canicule d'août 2003 ", Revue française de science politique, 55 (4), 2005.

7. Dossiers papier et informatique mais limités au ministère de l'Agriculture (aux échelons nationaux et locaux).
} 
d'août 2004 permet de mettre en lumière le rôle de cette nouvelle configuration qui, en opérant une brusque confrontation des pratiques professionnelles des vétérinaires et des médecins, en matière de recours aux médias dans la gestion de crise et d'utilisation de la vaccination dans la gestion des maladies, va fortement influer sur la transformation de cet épisode en crise.

\section{Le cas Tikki vu par les sciences sociales}

\section{De l'alerte sanitaire à la crise : récit d'une " crisogenèse "}

L'histoire du cas Tikki débute en juin 2004, au cours d'un séjour au Maroc, quand un jeune homme adopte une chienne, du nom de Tikki, et la ramène en France. Arrivé à destination, ce propriétaire, féru d'éducation canine, entreprend de socialiser son animal. Il multiplie ainsi les promenades dans Bordeaux et, durant la première quinzaine d'août, Tikki l'accompagne à trois reprises à des festivals de théâtre de rue en Aquitaine. Mais au cours d'une promenade, la chienne habituellement très affectueuse mord son propriétaire. Le comportement de Tikki change et elle traverse des accès de fureur qui contraignent son propriétaire à l'abandonner à la SPA où elle meurt et, compte tenu de ces symptômes, fait l'objet d'un prélèvement rage confirmé positif par l'institut Pasteur le 26 août 2004. Le jour même, le propriétaire est interrogé par les autorités sanitaires locales pour identifier les contacts animaux et humains de la jeune chienne. Une campagne de communication locale et nationale est orchestrée par les pouvoirs publics pour retrouver les personnes et les animaux ayant eu des contacts avec le chien enragé ${ }^{8}$. Un numéro de téléphone spécifique est immédiatement mis en place pour collecter des informations et répondre aux questions de la population ${ }^{9}$. En cas de contact avéré, les personnes sont envoyées dans un centre antirabique pour un traitement et les animaux sont euthanasiés. De leur côté, les services vétérinaires, accompagnés de la maréchaussée, mènent diverses enquêtes et reconstitutions sur les lieux de passage de l'animal. Ils complètent ainsi les informations sur les contacts de Tikki. Dès le 28 août, des arrêtés préfectoraux prescrivent sur le territoire des sept communes où la jeune chienne a circulé, des restrictions de circulation des chiens et des chats ${ }^{10}$ et l'abattage de tous les animaux errants sur ces communes. Après quelques jours, plusieurs contacts humains avérés de Tikki s'avèrent toujours introuvables (notamment un jogger et un couple accompagné de ses deux enfants). Le sujet prend une ampleur médiatique et la rage en Aquitaine fait l'ouverture du journal télévisé de France 2 à cinq reprises en l'espace de quinze jours (du 30 août au 13 septembre 2004). Le ministère de la Santé interroge alors celui de l'Agriculture sur l'opportunité de rendre obligatoire la vaccination antirabique

8. Trente-deux communiqués de presse entre le 28 août et le 15 septembre et deux conférences de presse.

9. Il recevra 3500 appels (source : communiqué de presse de la préfecture).

10. Les chiens ne peuvent circuler qu'en laisse et les chats dans des paniers fermés. 
des carnivores sur l'ensemble du territoire national ${ }^{11}$. Le ministère de l'Agriculture répond négativement à cette demande et préfere prendre une autre mesure radicale : il étend, par l'arrêté ministériel du 3 septembre $2004^{12}$, les mesures de restriction de circulation des carnivores et d'euthanasie des animaux errants à l'ensemble des communes des trois départements concernés. Mais, le 12 septembre, le ministre de la Santé, P. Douste-Blazy, en visite à la cellule de crise de la Préfecture de Bordeaux, annonce lors de la conférence de presse son souhait de voir la vaccination des carnivores rendue obligatoire sur l'ensemble du territoire national. Le ministre de l'Agriculture s'oppose à la mise en place de cette obligation de vaccination et met en avant les mesures de gestion prises par l'arrêté du 3 septembre. Les conséquences de cet arrêté contribuent pour beaucoup à la dynamique de la crise. L'ouverture de la chasse, date emblématique en Aquitaine, est par exemple compromise et les chasseurs sont condamnés à « chasser sans leurs chiens ", ce qui crée de nombreuses tensions locales ${ }^{13}$. Parallèlement, plusieurs centaines de chiens ayant échappé à la surveillance de leurs propriétaires s'accumulent dans les fourrières et se voient menacés d'euthanasie. Plusieurs groupes de particuliers ou des propriétaires attaquent les fourrières de Gironde et du Lot-et-Garonne pour libérer des animaux. Devant l'émotion de la population locale et nationale ${ }^{14}$, le préfet de Gironde annonce aux journalistes que les décisions d'euthanasie se feront au cas par cas, et ce sans l'aval du ministère de l'Agriculture et des comités d'experts scientifiques très frileux sur d'éventuelles dérogations à ces abattages. Au terme d'un long processus administratif pour donner un cadre juridique à ces dérogations, plusieurs milliers d'animaux seront mis sous surveillance et, selon les bilans officiels, trois cent deux chiens et chats seront euthanasiés, même si la rumeur circulant au sein des autorités fait état de mille deux cents euthanasies.

Ainsi à partir d'un événement relativement usuel, l'introduction d'un animal enragé en France, l'épisode de 2004 se caractérise pour les différents protagonistes interrogés par un emballement des processus médiatiques et gestionnaires qu'ils estiment typiques des crises. Loin de donner une définition normative d'une crise, nous adopterons ici le point de vue des acteurs, unanimes pour qualifier cet épisode de crise. Mais afin de caractériser cet emballement des processus, nous nous concentrerons sur les deux différences majeures entre ce cas et les précédents, à savoir l'orchestration d'une campagne de communication par les autorités et la radicalisation des mesures de gestion.

11. Quand la France n'était pas indemne de rage, la vaccination n'était obligatoire que dans les zones où la rage sévissait.

12. Arrêté du 3 septembre 2004 relatif à des mesures particulières de lutte contre la rage applicables dans les départements de la Dordogne, de la Gironde et du Lot-et-Garonne.

13. Réunions houleuses, désaccords entre services de l'État, attaque de la décision par la fédération départementale de la chasse de la Gironde notamment.

14. Dont témoignent environ une centaine de lettres de particuliers de toute la France à des parlementaires et au ministre de l'Agriculture. 


\section{La " crisogenèse " vue par les sciences sociales}

Pour rendre compte de ces processus d'amplification de certains problèmes publics, tout un courant de la sociologie des risques s'est concentré sur l'étude de la perception du risque par les individus, mettant ainsi en avant le rôle des représentations collectives dans la dynamique des crises ${ }^{15}$. Ces crises étranges ou inexplicables, selon les experts, pourraient être en partie le fruit d'une vision erronée du risque objectif par les profanes. Les crises seraient donc facilitées par une inquiétude disproportionnée de la population vis-à-vis d'une menace objectivement faible. Même si la rage constitue une maladie symbolique, fortement ancrée dans l'imaginaire collectif ${ }^{16}$, ce décalage ne suffit pas à rendre compte de la dynamique de cette crise. En effet, rien ne permet d'imaginer un changement de représentation de la maladie entre les trois cas très rapprochés de 2004 et d'expliquer ainsi la différence de traitement de ces épisodes. L'opposition entre une vision experte et une vision profane du risque n'apparaît donc pas comme la meilleure voie d'entrée pour expliquer notre phénomène.

Un autre courant de la sociologie des risques s'intéresse à la construction des crises à travers l'étude des médias ${ }^{17}$. Les " affaires " politiques, financières et sanitaires ont en effet favorisé l'émergence d'une nouvelle forme de journalisme d'investigation de nature polémique influant elle-même sur les dynamiques de l'action publique. Mais deux facteurs poussent à écarter l'origine médiatique comme seule grille de lecture de cette crise. Tout d'abord, les autorités communiquent de manière active en publiant presque deux communiqués de presse par jour ; ensuite, les médias soulèvent très peu de controverses dans cette couverture de l'événement. Sur les trois cents communications étudiées, seules quarante sept adoptent un ton polémique ${ }^{18}$ et un seul article dans le journal Le quotidien du médecin porte une controverse structurée en mettant clairement en cause l'action de l'État au sujet des défaillances du contrôle en frontière. Il est donc difficile d'expliquer ce phénomène par une forme de prise en otage des autorités par les médias.

Francis Chateauraynaud et Didier Torny se sont éloignés de ce modèle de la construction médiatique des affaires en centrant leur analyse sur les épreuves

15. Douglas (M.), Wildavsky (A.), Risk and Culture: An Essay on the Selection of Technological and Environmental Dangers, Berkeley, University of California Press, 1984 ; Wilde (G.), « The Theory of Risk Homeostasis: Implication for Safety and Health », Risk Analysis, 2, 1982.

16. Théodoridès (J.), Histoire de la rage, Paris, Masson, 1986.

17. Hermitte (M.), Le sang et le droit. Essai sur la transfusion sanguine, Paris, Seuil, 1996 ; Favre (P.), « L'émergence des problèmes dans le champ politique », in Favre (P.), dir., Sida et politique. Les premiers affrontements 1981-1988, Paris, L'Harmattan, 1992.

18. Nous avons considéré une communication comme "polémique » dès lors que les journalistes citent, sans les relativiser, ni les soutenir, des acteurs choisis pour leur désaccord ou leur hostilité à l'action des autorités sanitaires. Une communication a été considérée comme porteuse de controverse dès lors que l'auteur de l'article soutient clairement une position hostile à l'action des autorités. 
successives qui structurent le passage d'une alerte à la crise ${ }^{19}$. Ce modèle essentiel pour analyser l'accès progressif de nouveaux problèmes (amiante, prion, etc.) à l'agenda public n'est toutefois pas parfaitement adapté pour expliquer la soudaine mise en crise d'un problème public déjà fortement structuré comme la rage.

Nous nous inscrivons donc plutôt dans la lignée d'autres travaux de sociologie de l'action collective, qui considèrent le risque comme un construit social et technique et insistent sur le caractère souvent endogène des crises ${ }^{20}$. Ces travaux initiés dans le domaine des risques majeurs et des catastrophes industrielles ${ }^{21}$, puis élargis à l'analyse des " affaires", comme le SIDA ${ }^{22}$ et l'ESB ${ }^{23}$ ont contribué à étudier les crises comme des espaces nouveaux de contraintes et d'opportunités suscitées par la rencontre d'un événement déclencheur avec les groupes concernés. Ils ont principalement insisté sur les déficits organisationnels et cognitifs ou sur les effets de désectorisation ${ }^{24}$ liés à la rencontre de segments de la société traditionnellement étanches, qui doivent œuvrer ensemble au cours des phénomènes de crises. La crise, dont le caractère endogène est fortement souligné par ces travaux, est en partie liée à l'intervention simultanée de multiples organisations peinant à trouver une définition commune des problèmes rencontrés.

Les travaux de cette lignée plus spécifiquement tournés vers des crises du domaine sanitaire ${ }^{25}$ ont montré l'importance des reconfigurations de ce champ d'action publique dans les modalités de traitement des alertes sanitaires. Parallèlement, ils ont contribué à souligner l'impact de ces alertes ou crises dans la structuration des attributions de chacun dans ce nouvel espace institutionnel. Toutefois, nombre de ces travaux axés plus spécifiquement sur les concurrences

19. Chateauraynaud (F.), Torny (D.), Les sombres précurseurs. Une sociologie pragmatique de l'alerte et du risque, Paris, Éd. de l'EHESS, 1999.

20. Lemieux (C.), Barthe (Y.), « Les risques collectifs sous le regard des sciences du politique. Nouveaux chantiers, vieilles questions », Politix, 44, 1998 ; Borraz (O.), Gilbert (C.), Joly (P.), « Risques, crises et incertitudes : pour une analyse critique ", Cahiers du GIS Risques collectifs et situations de crise-MSH Alpes, 3, 2005.

21. Lagadec (P.), La civilisation du risque, Paris, Seuil, 1981; Lagadec (P.), La gestion des crises. Outil de réflexion à l'usage des décideurs, Paris, McGaw-Hill, 1991.

22. Setbon (M.), Pouvoirs contre Sida. De la transfusion sanguine au dépistage : décisions et pratiques en France, Grande Bretagne et Suède, Paris, Seuil, 1993 ; Dodier (N.), Barbot (J.), « Le temps des tensions épistémiques, le développement des essais thérapeutiques dans le cadre du sida ", Revue française de sociologie, 41 (1), 2000.

23. Alam (T.), «Crises, acteurs politiques et changement institutionnel, un nouveau système de gestion des risques alimentaires en Grande-Bretagne ", Revue internationale de politique comparée, 10 (2), 2003 ; Barbier (M.), « Une interprétation de la constitution de l’E.S.B. comme problème public européen », Revue internationale de politique comparée, 10 (2), 2003.

24. Dobry (M.), «Réflexions à partir d'une analyse sociologique des crises politiques ", Actes de la troisième séance du séminaire du programme risques collectifs et situations de crise, Grenoble, CNRS, 1995.

25. Besançon (J.), Borraz (O.), Grandclément-Chaffy (C.), La sécurité alimentaire en crises. Les crises CocaCola et Listéria de 1999-2000, Paris, L’Harmattan, 2004 ; Benamouzig (D.), Besançon (J.), " Administrer un monde incertain : les nouvelles bureaucraties techniques, le cas des agences sanitaires en France ", Sociologie du Travail, 47, 2005 ; Gimbert (V.), L'État sanitaire en question : les administrations à l'épreuve des risques, Thèse de doctorat, ENS Cachan, 2007. 
institutionnelles ont peu interrogé le rôle des pratiques professionnelles et de la confrontation de différentes solutions institutionnalisées de gestion des alertes dans l'émergence même des crises. Nous allons donc ainsi, dans la lignée de ces travaux qui replacent fréquemment les crises dans des perspectives à long terme, commencer par retracer le cadrage historique du problème rage en France.

\section{La rage en France : la carrière d'un problème public}

\section{8-1998 : Ie ministère de l'Agriculture s'approprie la rage}

La rage, disparue en France depuis 1941, réapparaît en 1968 via des renards allemands traversant les frontières de l'Est. Face à l'extension de la forme animale de la maladie, le ministère de l'Agriculture se saisit du problème et crée, en 1972, une Commission Interministérielle de Lutte (CIL) chargée d'élaborer un plan de lutte contre la rage. Cette structure réunit des représentants des ministères de l'Agriculture, de l'Intérieur, de la Santé publique, de la Protection de la nature et de l'environnement, de la Défense nationale, des Postes et Télécommunications et associe les laboratoires spécialisés comme l'institut Pasteur et le centre d'étude sur la rage de Nancy ${ }^{26}$. Le ministère de l'Agriculture, initiateur de la commission, désigne ses deux présidents successifs, tous deux de formation vétérinaire et choisis dans le giron du ministère ${ }^{27}$. Cette commission organise des réunions régulières (soixante-trois entre 1972 et 2005) et permet ainsi aux différents protagonistes de construire ensemble une politique de lutte. Ceux-ci développent une expertise commune et une façon partagée de concevoir la gestion de cette maladie. La rage est appréhendée sous son versant animal et la définition des modalités de gestion est très fortement imprégnée par un modèle de lutte contre les maladies animales issu de l'enseignement vétérinaire. Celui-ci est véhiculé par les présidents successifs de la commission et par les vétérinaires fortement représentés au sein de la $\mathrm{CIL}^{28}$. La commission, clairement pilotée par le ministère de l'Agriculture, devient rapidement le lieu d'échange interministériel où se construit la politique publique de la rage en France. Au cours de cette période, la rage, en dépit de sa gravité pour l'homme, s'impose comme un problème public piloté par le ministère de l'Agriculture.

\footnotetext{
26. Ce laboratoire est devenu plus tard l'AFSSA Nancy. Participent aussi à cette commission l'Office national des forêts, de la chasse, les fédérations françaises de protection de la nature, et la Fédération nationale des propriétaires forestiers et sylviculteurs. Cf. Toma (B.), « La commission interministérielle de lutte contre la rage ", Épidémiologie et santé animale, 48, 2005.

27. Respectivement un ancien chef des services vétérinaires français et un professeur de maladie contagieuse de l'École d'Alfort.

28. Les participants particulièrement impliqués dans la commission, à savoir le Président, le ministère de l'Agriculture et son bureau de la protection animale, l'institut Pasteur, le ministère de la Défense et le laboratoire de Nancy envoient majoritairement des représentants de formation vétérinaire à chacune des réunions.
} 
Cette appropriation est facilitée par les caractéristiques biologiques de la rage. Cette maladie virale mortelle commune à l'ensemble des mammifères se transmet par morsure : la contagion d'homme à homme n'a donc jamais été décrite. Ainsi, les animaux constituent-ils son réservoir exclusif, imposant l'idée que la lutte contre la rage chez l'homme passe essentiellement par la prévention et la lutte contre la maladie chez les animaux ${ }^{29}$. Le faible impact humain de la maladie au regard d'autres affections humaines peut aussi expliquer cette faible implication du ministère de la Santé.

Au final, la politique publique mise en place au cours de ces trente années de lutte reflète clairement l'asymétrie d'appropriation de ce problème public par les deux ministères concernés.

\section{Une politique publique asymétrique}

Les mesures de lutte contre la rage chez les animaux sont définies par un ensemble de dispositions réglementaires rassemblées dans le code rural. Le ministère de l'Agriculture via la Direction générale de l'alimentation (DGAL) et ses services de terrain, les directions départementales des services vétérinaires (DDSV), est chargé de la conception et de l'application de ce corpus réglementaire. Le code rural encadre essentiellement le fonctionnement d'un réseau de surveillance : les vétérinaires sont chargés de repérer au plus tôt les animaux suspects d'être enragés. En cas de confirmation de la présence du virus rabique, un ensemble de mesures de lutte est activé ${ }^{30}$. Il prévoit la mise en œuvre d'actions sévères et coercitives telles que l'abattage de tous les animaux susceptibles d'avoir côtoyé l'animal enragé. La réglementation prévoit aussi la limitation des rassemblements d'animaux (foires, concours, expositions, chasse), de sévères restrictions à la circulation des chiens et chats et l'abattage dans les fourrières de tous les animaux errants capturés dans la commune de découverte du cas.

Le volet humain de la lutte contre la rage consiste à soigner les hommes susceptibles d'avoir été contaminés par un animal enragé. Du fait de la lente propagation du virus dans l'organisme humain, le vaccin rabique s'avère efficace, même s'il est injecté après le contact contaminant ${ }^{31}$. L'homme peut donc être traité après une morsure ou un contact. Le volet humain est ainsi structuré autour de mesures sans caractère contraignant, qui consistent à inciter toute personne en contact avec un animal suspect ou contaminé par la rage à se rendre au sein d'un centre hospitalier pour être traité dans un centre antirabique avant l'apparition des premiers symptômes de la maladie. Il s'agit donc d'une

\footnotetext{
29. Une approche alternative, plus axée sur la santé humaine, aurait pu cadrer le problème différemment en insistant plus sur la prévention des contaminations des voyageurs lors de leur séjour à l'étranger (vingt-trois cas décrits entre 1973 et 2003).

30. Décrit par l'AM du 6 février 1984.

31. C'est donc un des rares vaccins de nature curative et pas simplement préventive.
} 
politique qui correspond plus aux attributions de la médecine curative hospitalière qu'à une politique de prévention collective pilotée par le ministère de la Santé. Ce ministère, via la Direction générale de la santé (DGS) ${ }^{32}$, officiellement chargée d'instruire les rares dossiers d'ouverture de centres antirabiques au sein des hôpitaux, a délégué le pilotage de ces centres à l'institut Pasteur.

Ce double dispositif de lutte contre la rage est donc fondé sur l'association de deux logiques et organisations différentes. Le ministère de l'Agriculture prône une logique axée sur la surveillance et la prévention de la maladie chez l'animal, mobilisant des outils réglementaires et des mesures coercitives pilotées étroitement et directement par ses propres services. Pour les autorités de la Santé, la logique est curative et incitative, elle s'appuie sur la mise à disposition des moyens de traitement pour les personnes contacts qui le souhaitent, et cette politique est gérée en dehors du ministère par le réseau de centre antirabique animé par l'institut Pasteur. Si ces deux versants sont fondamentaux et concourent tous deux à la politique publique de lutte contre la rage, la gestion objective de la maladie et le développement de savoir-faire administratifs dans le domaine des concepteurs de politique publique sont surtout présents, historiquement, au sein du ministère de l'Agriculture.

\section{La progressive "ringardisation » de la rage : une politique publique hors agenda}

À partir de 1988, la CIL, sous l'impulsion de son deuxième président, un professeur de maladies contagieuses de l'école vétérinaire d'Alfort, joue un rôle décisif dans la mise en place d'un plan ambitieux d'éradication de la maladie chez les renards. Les membres de la CIL réalisent un travail de lobbying efficace pour obtenir les financements nécessaires à la réalisation de campagnes de vaccination orale des renards ${ }^{33}$. Il s'agit d'organiser le largage par hélicoptères d'appâts vaccinaux au-dessus des forêts afin de protéger les populations vulpines en zone contaminée. La stratégie d'éradication mise en place fonctionne et le virus disparaît du sol français, le dernier cas de rage étant recensé en 1998, la France est déclarée indemne de rage en 2000 par l'office international des épizooties.

Mais l'efficacité de la politique publique d'éradication conduit parallèlement à la perte d'intérêt stratégique de cette politique. Les problématiques résiduelles abordées en commission intéressent de moins en moins les protagonistes historiques du dossier. Peu à peu, les réunions s'espacent (en moyenne : quatre réunions par an de 1972 à 1976, puis deux réunions par an jusqu'en 1996, une réunion par an de 1997 à 2005) et en l'absence de problèmes importants à

32. Plus spécifiquement la sous-direction de la prévention des risques infectieux.

33. Toma (B.), « La commission interministérielle de lutte contre la rage », art. cité. 
résoudre ensemble, les liens entre acteurs se distendent jusqu'à la dissolution officielle de la CIL prononcée en 2005, peu après la crise d'août 2004, par la Direction générale de l'alimentation (DGAL) ${ }^{34}$.

« Le travail entre membres de la CIL se passait assez bien [...] car on savait où l'on allait! Il y avait un président pour diriger le débat. Il était légitime [...]. Petit à petit avec l'émergence de l'AFSSA et des comités d'experts spécialisés, de l'InVS, puis l'absence de cas depuis 1998 on s'est rendu compte que le comité interministériel n'avait plus lieu d'être ; c'était devenu une instance très technique qui gérait vraiment des petites décisions très spécifiques qui moi ne m’intéressaient pas. » (Vétérinaire, institut Pasteur)

Au cours de cette période, il n'est plus question de construire une politique de lutte ambitieuse mais simplement d'appliquer des mesures de gestion codifiées aux rares cas qui se présentent sur un territoire indemne (importations d'animaux de pays interdits, traitement suite à des retours de touristes mordus en zone contaminée, analyses d'encéphales d'animaux suspects, etc.). La rage apparait alors comme une problématique sanitaire secondaire.

\section{La rage prête à renaître dans une nouvelle configuration}

\section{Les leçons de la canicule : la réhabilitation des vieux risques}

Entre 1996 et le déclenchement du cas Tikki, en août 2004, la succession des crises sanitaires avec le SIDA tout d'abord, l'ESB ensuite, et en dernier lieu la canicule, entraîne une profonde évolution du contexte organisationnel de la gestion de la sécurité sanitaire : réorganisation de la Direction générale de la Santé (DGS) et de la Direction générale de l'alimentation (DGAL), création de l'Agence française de sécurité sanitaire des aliments (AFSSA) ${ }^{35}$ et de l'Institut de veille sanitaire (InVS), etc.

Les évolutions consécutives aux crises sanitaires n'affectent pas directement la gestion du dossier rage, qui n'est pas sous les feux des projecteurs et reste confinée à des problématiques techniques. Cependant, suite à la canicule de 2003, de sévères critiques ont été formulées vis-à-vis de la DGS et de l'InVS, accusés de ne pas avoir donné l'alerte ${ }^{36}$. La canicule a ainsi profondément réorienté la perception de ce qu'est une crise sanitaire. Après la canicule, les critères d'appréciation de la gravité d'une alerte s'élargissent. Aux problèmes d'émergence de

\footnotetext{
34. La DGAL annoncera en 2005 l'intégration de la CIL au sein du comité consultatif de la santé et protection animale.

35. Nous n'évoquerons pas en détail ici les reconfigurations de ce champ liées à la création de l'AFSSA (qui englobe le laboratoire national de référence rage de Nancy) qui remodèle en profondeur les modalités d'accès à l'expertise scientifique en matière de rage pour le ministère de l'Agriculture.

36. Lagadec (P.), Laroche (H.), Retour sur les rapports d'enquête, op. cit., p. 29.
} 
nouvelles pathologies confrontant les gestionnaires à l'insuffisance des savoirs scientifiques (ESB, antennes relais, etc.) viennent s'ajouter des « vieux risques » pourtant bien connus. L'accent est mis sur la capacité des systèmes à prendre en charge les risques, quels qu'ils soient, plus que sur une catégorie particulière de risques ${ }^{37}$. La canicule sert donc de précédent permettant à des problèmes publics connus d'être caractérisables ou pensables en termes de crise.

Dans ce cadre renouvelé, tout événement de santé, même historique et bien connu, doit conduire à un engagement fort des autorités dès lors qu’il dépasse une certaine ampleur.

« À l'époque, après le départ du DGS, on était juste après la canicule. Le nouveau DGS, il avait un petit peu peur de tout. C'est quelqu'un, en tant que DGS, qui voulait qu'on soit toujours, tout de suite dans l'action, au cas où ! Il était marqué par la canicule, $[\ldots]$ il n'avait pas la culture de l'alerte et il avait toujours peur que tout événement de santé parte en vrille, il fallait de suite agir !» (Contractuel, InVS)

On peut faire l'hypothèse que cet engagement dans une «crise » est d'autant plus fort que les organisations concernées ont été dans un passé récent mises en défaut sur la prise en charge d'un épisode. La volonté collective des organisations de la Santé de se distinguer par une politique réactive au lendemain d'une remise en cause violente liée à la canicule de l'été 2003 apparaît comme un facteur important de la forte réactivité des autorités publiques face au cas de rage de l'été 2004.

Cet engagement fort dans la « prise en charge » des crises se traduit notamment en termes organisationnels par la création du département des urgences sanitaires (DUS). Le DUS, directement rattaché au directeur général, est chargé de toutes les alertes qui dépassent les possibilités de gestion à l'échelon local. Cette nouvelle entité répond à un besoin, perçu par la DGS, de professionnalisation de la gestion de l'urgence face à la succession des crises et à la complexification des missions assumées.

«À partir de juin 2004 en gros, on a eu une évolution sur le bureau des alertes qui est devenu un département des situations d'urgence sanitaire qui s'est étoffé d'un pôle responsable de la gestion et de l'acquisition des stocks nationaux de matériels et de Tamiflu... D'un seul coup, on a vu apparaitre effectivement des administratifs, des gens qui étaient ou logisticiens ou juristes, ou économes, ou administrateur civil, ou attaché d'administration centrale, parce que l'on avait de plus en plus de stocks à gérer, à acheter, à acquérir [...]. Donc l'alerte, elle est tombée en plein cela car la création officielle de ce département, c'est par

37. Coquin (Y.), Dab (W.), Laurens-Bernard (O.), « Les enjeux de santé publique de la gestion des risques et des crises ", Gestions hospitalière, 439, 2004. 
l'arrêté du 15 septembre 2004, c'est lié à la fois aux suites de la canicule et au constat qu'en gros un bureau technique ne pouvait pas s'occuper bien, ou correctement, et de manière professionnelle, de tout ce qui était l'acquisition des stocks, par exemple, car c'est un vrai métier. » (Pharmacien inspecteur, DUS)

Cette première modification organisationnelle, en cours au moment de l'épisode Tikki, facilite le processus de renégociation de la propriété de ce problème public en introduisant, via le DUS, de nouveaux acteurs non familiarisés avec le rôle de leader acquis par le ministère de l'Agriculture en matière de gestion du problème public rage.

Parallèlement, la création de l'InVS par la loi de 1998 joue un rôle similaire. Placé sous la tutelle du ministère de la Santé, l'institut est un établissement public chargé des missions de surveillance, de vigilance et d'alerte dans tous les domaines de la santé publique. L'InVS est chargé de désigner les centres nationaux de référence (CNR), dont fait partie le Centre national de référence sur la rage (on le désignera pour la suite sous l'appellation globale d'institut Pasteur). Au lendemain des mises en cause liées notamment à la canicule, l'InVS a vu ses missions complétées et renforcées par la loi du 9 août 2004 relative à la politique de santé publique. Cette modification législative intervient donc quelques semaines à peine avant la crise d'août 2004 et elle rajoute aux missions de veille de l'InVS la contribution à la gestion des situations de crises sanitaires. Dans une politique publique dont le versant humain, délaissé par le ministère de la Santé est essentiellement géré par l'institut Pasteur, la création de l'InVS assurant sa tutelle et titulaire de prérogatives en matière de gestion de crise va donc modifier l'équilibre institué entre les agents des ministères de l'Agriculture et de la Santé.

Ainsi, à l'aube de la crise d'août 2004, les créations de l'InVS et du département des urgences sanitaires remettent en cause le contexte ayant permis « l'appropriation » historique de la problématique rage par le ministère de l'Agriculture et l'équilibre du réseau d'acteurs gestionnaires. Elles réintroduisent des possibilités d'implication du ministère de la Santé dans le dossier rage. Parallèlement, au lendemain de la canicule, le succès de la prise en charge d'un épisode sanitaire apparaît pour l'InVS et la DGS comme un enjeu susceptible de réhabiliter leur action. Toutefois, cette perspective organisationnelle et stratégique n'épuise pas l'explication du phénomène. Selon cette grille de lecture, on aurait pu s'attendre à ce que, par exemple, le premier cas de rage de 2004, soit saisi par les autorités et fasse l'objet d'un traitement radicalement nouveau. Pourtant, seul le troisième cas de rage de 2004 va passer du statut d'alerte à celui de crise. Si l'on exclut une perspective d'explication fonctionnaliste associée à la forte présence de personnel technique d'astreinte en ce mois d'août, anniversaire de la canicule de 2003, on peut éclairer ceci en observant comment le nouveau dispositif institutionnel s'est confronté aux épisodes successifs de rage. 


\section{Une mise à l'épreuve graduelle du nouveau dispositif}

Fin 2003, juste quelques mois après l'épisode canicule, un cas de rage met à l'épreuve le nouveau système de gestion des risques sanitaires. Contrairement à 1968 où la rage est réapparue sous sa forme animale, cette fois-ci il s'agit d'un cas humain, un petit garçon contaminé par un chiot lors d'un séjour au Gabon. En l'absence de contagion interhumaine décrite en matière de rage, le problème aurait pu rester de l'ordre des drames courants de la médecine individuelle. Toutefois, l'InVS, marqué par la canicule, se saisit du cas et organise une intervention massive de médecins chargés d'informer et de rassurer les parents d'élèves de la maternelle fréquentée par le petit garçon.

«Il faut savoir que cela [le cas d'août 2004] intervient dans un contexte, l'année précédente, il y avait eu un cas humain importé qui est mort évidemment, qui était un gamin et que cette crise-là, la gestion a été très compliquée, sur le gamin, c'était très très compliqué car cela s’est passé juste à la fin de la canicule donc à un moment où on était tous un petit peu secoués par la presse et là-dessus, il y a eu ce cas humain qui ne s'est pas bien passé, donc ça c'était fin 2003.» (Contractuel, InVS)

S'agissant d'un cas humain, le ministère de l'Agriculture n'est pour ainsi dire pas associé à sa gestion alors que les chargés du dossier, au ministère de la Santé et surtout à l'InVS, développent leurs savoirs théoriques et pratiques en matière de rage, en consultant notamment les données scientifiques disponibles sur la maladie et en se confrontant aux problèmes pratiques posés par cette expérience ${ }^{38}$.

De surcroît, les parutions scientifiques émanant de l'institut Pasteur qui font état d'un risque émergent de rage par le biais des chauves-souris ont poussé le ministère de la Santé à solliciter le Conseil supérieur d'hygiène publique de France (CSHPF) qui a crée deux groupes de travail sur la thématique rage. Courant 2003-2004, les acteurs institutionnels du dossier de la rage ont ainsi été amenés à participer à ces groupes de travail, dont le pilotage a été délégué à l'InVS par le $\mathrm{CSHFP}^{39}$. La conjonction du cas humain et de ces groupes de travail ont ainsi réactivé des contacts, redonné une visibilité à cette problématique dans la sphère de la santé publique et développé les connaissances sur la rage au sein du ministère de la Santé, jusque-là peu concerné.

« L'institut national de veille sanitaire a donc acquis cette compétence nouvelle liée aussi à la présence de [nom du chargé de dossier] qui a mené des groupes et a vraiment développé des compétences en matière de rage au cours des années 2003 et 2004. À la Direction générale de la Santé, il y avait donc à ce moment-là les compétences en matière de rage. » (Vétérinaire, institut Pasteur)

38. Notamment la gestion de la communication vis-à-vis des parents, l'organisation d'une vaccination en masse, et la difficulté de restreindre ces vaccinations du fait d'avis divergents sur les risques pour le personnel hospitalier.

39. Environ cinq réunions et de nombreux échanges d'e-mails. 
C'est dans ce contexte en pleine mutation que s'inscrit, en janvier puis en mai 2004, la découverte de deux chiens enragés en provenance du Maroc (respectivement dans le Morbihan et en Gironde). Ces deux premiers cas passent inaperçus en dehors des organisations gestionnaires ${ }^{40}$ mais sont l'occasion pour les autorités de la santé d'approfondir les contacts et de se familiariser avec les pratiques du ministère de l'Agriculture en matière de gestion de cas de rage chez l'animal. En ce qui concerne le ministère de l'Agriculture, les vétérinaires du bureau en charge de la rage à la DGAL évoquent aussi cet effet d'apprentissage et une gradation dans les trois alertes rage de 2004 : la première a permis de réviser les textes et les données sur cette maladie et de mettre en œuvre des solutions et la seconde de tester et confirmer ces savoirs théoriques et pratiques. "Donc je dirais que ce deuxième cas c'était une répétition, un échauffement, de ce qui allait arriver en août à bien plus large échelle ${ }^{41}$. »

Au moment de la crise d'août 2004 qui prend racine dans le même département que le second cas, les acteurs gestionnaires centraux et locaux du ministère de l'Agriculture sont restés au même poste. Ils se sentent donc parfaitement rodés à la gestion d'un épisode de rage.

"À la limite, cela devenait de plus en plus intéressant et exaltant car comme cela avait parfaitement bien fonctionné et que l'on savait parfaitement faire fonctionner la machine sur le terrain et ici, on se disait que c'était un troisième cas où l'on pensait qu'on pourrait faire encore mieux ce que l'on avait déjà fait très bien. » (Vétérinaire, DGAL)

Cette remise à niveau progressive des savoirs administratifs au gré des épisodes de 2003 et 2004 nous éclaire sur le processus de passage de l'alerte à la crise en août 2004. Au sein du ministère de l'Agriculture, cette transition peut être favorisée par la levée des freins liés à l'appréhension d'une situation inconnue. Les agents du ministère de l'Agriculture traditionnellement à l'aise avec cette maladie, du fait de la répétition rapprochée des cas, maîtrisent parfaitement le dispositif et craignent peu d'être pris en défaut dans leur gestion. Pour les autorités de la santé, les bouleversements organisationnels, susceptibles de rompre l'asymétrie d'appropriation historique entre ministères, ne prennent pleinement forme qu'après développement et rééquilibrage des savoirs relatifs à la rage. Ce double processus relatif aux organisations et aux savoirs met les acteurs de la Santé en position de pouvoir renégocier le compromis historique de gestion de la rage. C'est ainsi que les approches et les pratiques différentes des deux ministères en matière de lutte contre les maladies vont pouvoir jouer et influer directement sur la dynamique de la transformation de l'alerte en crise.

40. Aucune médiatisation nationale et respectivement une et deux conférences téléphoniques interministérielles, contre onze dans l'épisode Tikki.

41. Vétérinaire, DGAL. 


\section{Tikki ou l'affrontement de deux modèles de gestion des maladies}

\section{Le cas Tikki}

Le 26 août 2004, dès l'annonce par l'institut Pasteur d'un résultat rage positif sur une jeune chienne prélevée dans une SPA et originaire du Maroc, la DGAL insiste pour avoir au plus tôt le maximum d'informations sur les contacts de l'animal. Le propriétaire est interrogé le soir même pendant plus de trois heures dans les locaux de la DDSV afin de reconstituer les faits. Les agents du DUS décident de l'organisation dans l'après-midi même d'une réunion interministérielle de « crise » dans les locaux du ministère de la Santé. Il s'agit là d'une procédure exceptionnelle : dans les épisodes précédents, la concertation avait eu lieu essentiellement par échanges téléphoniques entre les protagonistes habituels du dossier rage. Cette première "réunion de crise » regroupe des agents de niveau hiérarchique variable en fonction des institutions. On retrouve pour les autorités de la Santé des niveaux hiérarchiques élevés témoignant de l'importance donnée en moins de vingt-quatre heures à cet événement ${ }^{42}$, alors que les représentants du ministère de l'Agriculture sont d'un niveau hiérarchique nettement inférieur ${ }^{43}$. À ce stade précoce, on observe donc un très fort repositionnement des acteurs de la Santé dans le dossier rage qui, via le DUS et l'implication des hiérarchies des différents organismes, prennent en main cet événement sanitaire. Ce constat vient donc étayer l'hypothèse, précédemment développée, du rôle décisif des évolutions organisationnelles, stratégiques et cognitives dans la gestion du cas Tikki. Mais comment ces évolutions vont-elles participer à transformer cet événement en crise ? Nous privilégierons, pour éclairer cette question, deux préoccupations majeures des acteurs qui témoignent selon eux de l'emballement des processus médiatiques et de gestion : l'ampleur des recherches de personnes et des chiens en contact avec Tikki et les débats autour de la vaccination. Ces deux sujets constituent aussi les deux thèmes majeurs traités par la presse au cours des quinze premiers jours de cet épisode, conditionnant ainsi la vision de cette crise dans l'espace public.

\section{De l'enquête épidémiologique à l'appel aux médias : la reconfiguration de l'ampleur du cas Tikki}

Les protagonistes de l'épisode Tikki expliquent l'ampleur de ces recherches de contacts et le retentissement particulier de cet épisode par le caractère exceptionnel de l'événement sanitaire déclencheur. Il y aurait, selon eux,

42. Assistent à cette première réunion, un conseiller technique du ministre, la sous-directrice des risques infectieux, deux agents du DUS et de la cellule communication, le directeur de l'InVS, le directeur du laboratoire rage de l'institut Pasteur.

43. Seul un chef de bureau protection animale de la DGAL et un chargé de mission sont présents. 
une corrélation étroite entre les données épidémiologiques existantes sur le cas Tikki et son impact. Sans nier la conjonction de caractéristiques épidémiologiques étayant la dangerosité du cas (long séjour de l'animal sur le territoire français en période potentielle d'excrétion, nombreux déplacements et contacts de l'animal, notamment sur les sites de trois festivals), celles-ci ne suffisent pas à rendre compte pleinement des spécificités de la gestion du cas Tikki.

En effet, le propriétaire au cours de son « audition » décrit très précisément les personnes et les animaux avec lesquels Tikki a eu un contact rapproché pendant la période contaminante. Le nombre de contacts officiellement déclaré par les autorités locales est ainsi de six personnes et d'une dizaine de chiens ${ }^{44}$, soit un nombre du même ordre que dans les deux cas précédents de $2004^{45}$. Pourtant, un décalage s'instaure entre le faible nombre de contacts désignés par le propriétaire et le nombre de contacts potentiels, que les autorités sanitaires estiment à plusieurs milliers. Celles-ci évoquent ce cas comme un cas exceptionnel du point de vue du nombre de contacts, certains organismes comme l'InVS pouvant avancer le chiffre de 60000 contacts, correspondant à l'ensemble des personnes ayant assisté aux trois festivals où Tikki a été présente.

« Le vendredi, on apprend que la semaine d'avant il avait été avec son chien dans un festival de musique en plein air, donc sans listes de personnes présentes, Woodstock quoi! Donc un chien enragé lâché dans Woodstock. Et là, on s'est dit : "là, pas bon !" Et en creusant un peu, la semaine d'avant, il y avait eu un autre festival et puis encore un autre. Donc rapidement on s'est mis à chercher 60000 personnes en tout. Et là on s'est dit : "pas bon du tout" !” (Vétérinaire, InVS)

La différence notable entre ce cas et les précédents, différence qui établit une rupture nette entre eux, tient essentiellement au fait que les autorités sanitaires ne vont pas, cette fois, se limiter à rechercher les contacts humains et animaux identifiés par le propriétaire de l'animal enragé, mais dimensionner les recherches sur l'ensemble des contacts théoriquement possibles.

Au vu de l'accent mis dans les discours sur le caractère marginal du propriétaire de l'animal, on peut trouver là une première hypothèse pour rendre compte de ce changement dans l'évaluation du nombre de contacts. Le jeune propriétaire est régulièrement qualifié, par des acteurs n'ayant eu aucun contact avec lui, de "désocialisé » (un médecin inspecteur), de "saltimbanque » (un administrateur en Préfecture), de " hippie, de vagabond, de marginal, d'artiste de rue » (inspecteurs de santé publique vétérinaire), de «SDF » (médecin

\footnotetext{
44. Sources : rapport d'audition du propriétaire.

45. Le rapport de la DDSV 56 suite au cas de février fait état en effet de 23 personnes contact dont 12 dans l'entourage immédiat du propriétaire et de la fugue du chien en pleine ville (Lorient) pendant deux heures en pleine période de symptômes et d'excrétion salivaire.
} 
inspecteur, chasseur, technicien des services vétérinaires), les festivals de théâtre de rue deviennent le festival de "Woodstock ${ }^{46}$ et les références à la drogue sont très nombreuses dans les discours des autorités. Les gestionnaires du risque à l'échelon national relient les caractéristiques sociales du propriétaire et leurs conséquences sur le comportement de son animal. Tikki est immédiatement suspectée d'avoir été laissée sans surveillance et en constante divagation alors que le propriétaire assure, quant à lui, avoir gardé sa chienne en permanence sous son contrôle ${ }^{47}$. La marginalité assignée au propriétaire de Tikki pourrait donc en principe expliquer ce changement.

Pourtant, au niveau local, chez les agents des DDSV qui sont les seuls à avoir eu plusieurs entretiens directs avec l'intéressé, si la rhétorique de la «marginalité » n'est pas absente, elle n'est pas dominante et les discours insistent plutôt sur les éléments visant à crédibiliser les déclarations de ce propriétaire : il est question de son diplôme de maîtrise, de son exceptionnelle mémoire au sujet des contacts de son chien, de sa très bonne connaissance de l'éthologie canine, de sa réelle surveillance des activités de son animal et de son implication pour aider à retrouver les contacts de Tikki.

" Il faut dire que l'on sentait aussi que le gars au niveau de l'enquête, il avait vachement bien cadré, le gars avait beaucoup de mémoire et puis vraiment précise et il était tout le temps avec son chien, son chien ne s'était pas trop barré, il s'était baladé mais toujours avec son chien. C’est quelqu'un qui est passionné d'animaux et qui est très attentif, cela va faire un excellent dresseur. » (Vétérinaire, DDSV)

Ce désaccord entre le niveau central piloté en interministériel mais sur lequel la Santé a pris la main et le niveau local où le ministère de l'Agriculture via ses services de terrain (DDSV) prédomine, pose question. Cette nouvelle façon d'estimer le nombre de contacts est-elle vraiment liée à des facteurs sociaux qui viendraient affaiblir la confiance dans les déclarations du propriétaire ? Pourrait-elle s'expliquer plutôt par des pratiques différentes de la gestion de ce type d'événement sanitaire entre les acteurs de la Santé et de l'Agriculture?

Lors des deux précédents cas de 2004, les propriétaires étaient déjà décrits comme des marginaux, montrant que la rupture dans cet épisode ne se situe pas spécifiquement sur ce point.

«C'étaient des jeunes qui avaient été [...] dans des communautés hippies libertaires, des chevelus avec des petites fleurs dans les cheveux, etc., donc des gens un petit peu bohèmes, avec des chiens évidemment pas attachés.» (Vétérinaire, DGAL)

\footnotetext{
46. Pourtant le jeune homme, certes sans emploi, dispose d'un appartement dans le centre ville et est titulaire d'un diplôme de maîtrise d'informatique appliquée à la gestion des entreprises. 47. À l'exception de quelques minutes, un matin sur le parking d'un festival.
} 
Les références à la marginalité, si présentes au cours de l'épisode Tikki, peuvent être regardées aussi bien comme l'expression d'une rupture de confiance vis-à-vis des déclarations du propriétaire que comme une technique de légitimation d'une soudaine révision du mode d'estimation du nombre de contacts qui aurait d'autres causes.

On peut en effet rendre compte de cette évolution par la soudaine confrontation, au cours de cet épisode des pratiques des nouveaux acteurs en présence. Revenons donc aux pratiques de gestion de la rage employées par les agents des DDSV. L'identification des contacts de l'animal enragé a traditionnellement lieu par le biais d'un outil particulier et habituel dans le cadre des maladies animales : l'enquête épidémiologique. Elle consiste à mener des investigations sur le terrain afin d'identifier avec le maximum de certitudes les circuits des animaux impliqués dans la maladie. La mise en œuvre d'une telle technique, proche des investigations policières ${ }^{48}$, est fortement influencée par la finalité des recherches opérées qui visent la mise en œuvre de mesures coercitives. Dans ce contexte, lorsqu'il s'agit de bloquer des élevages ou de mettre à mort des animaux, le recours aux médias ou à la simple déclaration volontaire des propriétaires d'animaux contacts s'avère souvent inefficace. Dans les cas de rage précédents, les services vétérinaires s'étaient donc reposés sur les déclarations du propriétaire de l'animal enragé, identifiant lui-même les contacts, tout en procédant à des recoupements ou à diverses investigations ${ }^{49}$. Les gestionnaires du risque avaient donc considéré, jusque-là, que le propriétaire était à même de fournir une liste presque exhaustive ou au moins une description d'une grande partie des personnes et animaux avec lesquels l'animal enragé avait été en contact. Dans le cas Tikki, le recours aux médias viendra se superposer à l'enquête épidémiologique (qui restera toutefois la base de travail des agents de terrain).

Cet appel aux médias est courant dans le cadre des alertes relatives à la santé humaine où l'on recherche généralement des personnes pour les soigner. Ce recours aux médias, en tant qu'auxiliaires de service public, n'est pas neutre. Il entraîne un brutal redimensionnement de l'alerte et fait passer cet épisode du modèle traditionnel de gestion confinée à la sphère professionnelle à un nouveau modèle de confrontation dans un espace public ${ }^{50}$.

Ce qui rend le cas Tikki si différent, et peut amener à parler de 60000 contacts potentiels, c'est donc moins le cas en soi, que la remise en cause brutale, du fait

48. Berdah (D.), «Suivre la norme sanitaire ou "périr" : la loi de 1954 sur la prophylaxie collective de la tuberculose bovine », in Bonneuil (C.), Denis (G.), Mayaud (J.-L.), dir., Sciences, chercheurs et agriculture : Pour une histoire de l'agronomie, Paris, L'Harmattan, 2008.

49. Ainsi, dans le cas de mai 2004, les agents des services vétérinaires avaient retrouvé une dame et son chien qui avaient croisé l'animal enragé au cours d'une promenade au bord d'un lac, en refaisant eux-mêmes le trajet du propriétaire pour retrouver le lac en question et en interrogeant tous les résidents du bord du lac. 50. Torny (D.), «La traçabilité comme technique de gouvernement des hommes et des choses », Politix, 44, 1998. 
du nouveau jeu organisationnel, d'une estimation du nombre de contacts basée sur une enquête épidémiologique. Au regard de cette analyse, l'ampleur du cas Tikki apparait liée plus à l'introduction d'une nouvelle pratique de santé publique (recours aux médias) qu'à son caractère intrinsèquement exceptionnel.

Mais, si la superposition de ces deux pratiques (enquête épidémiologique, recours aux médias) contribue à créer un effet de rupture, à redimensionner l'épisode et à le façonner en crise, en pratique, ces deux visions restent superposables. En revanche, cela ne sera pas le cas en ce qui concerne la définition et le paramétrage des mesures à mettre en œuvre pour prévenir l'extension d'un risque défini de façon si large par cette nouvelle manière de concevoir le nombre de personnes exposées au risque. Une deuxième confrontation, plus violente, va donc avoir lieu autour de la question : faut-il vacciner contre la rage l'ensemble des carnivores français?

\section{Vaccination contre euthanasie : les conséquences de l'affrontement de deux modèles dans la dynamique de la crise}

Les deux ministères en présence ont une vision très différente du recours à la vaccination. Au ministère de la Santé, une partie de l'action de santé publique a historiquement porté sur la lutte pour une meilleure mise en œuvre des obligations de vaccination. Même si l'heure est aujourd'hui à la recommandation vaccinale, la culture historique en France dans le cadre de la lutte contre les maladies humaines est celle de l'obligation vaccinale ${ }^{51}$. Quand un vaccin existe, s'avère efficace, sans danger, et que le coût est raisonnable compte tenu de l'impératif de santé publique visé, la politique a longtemps été de le rendre obligatoire. Du côté des services de l'Agriculture conformément aux pratiques traditionnelles de lutte contre les maladies animales, la vaccination, qui dans de nombreuses maladies ${ }^{52}$ empêche le diagnostic, n'est pas considérée comme la solution la plus appropriée. La vaccination constitue une technique employée dans les phases précoces de lutte quand la maladie est fortement implantée sur un territoire et qu'il est difficile d'abattre tous les animaux contaminés. La solution historique qui consiste à éradiquer les maladies par des pratiques d'abattage et d'isolement, est toujours préférée lorsqu'elle est possible ${ }^{53}$. Être reconnu indemne d'une maladie sur un territoire et pouvoir s'en prévaloir au regard des normes internationales implique presque toujours d'interdire la vaccination contre cette maladie ${ }^{54}$. On a donc, d'un côté, une culture construite autour de l'obligation vaccinale et, de l'autre, une culture orientée vers l'interdiction vaccinale.

51. Bertrand (A.), Torny (D.), «Libertés individuelles et santé collective », Rapport du Centre de recherche médecine, sciences, santé et société (CERMES), 2004.

52. Dont la rage ne fait pas partie.

53. Collectif des professeurs de maladies contagieuses des écoles vétérinaires françaises, Polycopié de réglementation sanitaire générale, mise à jour informatique 2007.

54. Ceci afin que la vaccination ne dissimule pas la circulation de l'agent pathogène à bas bruit dans la population animale. 
Dés le début de l'épisode Tikki et le constat de son fort retentissement médiatique, cette tension se fait sentir. Certains acteurs au sein de la hiérarchie de la DGAL s'interrogent sur le recours aux pratiques vaccinales chez l'animal comme instrument de gestion de crise. L'éventualité d'une obligation de vaccination des carnivores est envisagée au sein du ministère de l'Agriculture, comme une mesure plus politique que technique qui permettrait de "rassurer » la population, de montrer l'action de l'État et de répondre aux sollicitations du ministère de la Santé. Mais cette idée heurte fortement les vétérinaires en charge au quotidien du dossier de la rage, pour qui l'obligation vaccinale n'est concevable qu'en dernier recours après avoir épuisé toutes les autres solutions disponibles. Cette éventualité vaccinale est donc provisoirement écartée.

"Quand on a vu que cela prenait une ampleur médiatique importante, c'est moi qui ai proposé de réfléchir à une vaccination des chiens. Au départ, c'est moi qui ai dit: "il faut que l'on fasse quelque chose car sinon on va se faire massacrer par la Santé, il faut qu'on mette en place quelque chose qui à la fois peut avoir une efficacité technique"; mais bon on ne voyait pas bien l'efficacité technique de la vaccination mais on voyait bien l'efficacité médiatique. Moi, j'étais sur l'idée de rendre la vaccination obligatoire et quand j'en ai parlé à [Nom d'un acteur de la hiérarchie] et je lui ai dit qu'on pourrait peut-être rendre obligatoire la vaccination sur tout le territoire français, on a réfléchi, on s'est dit que c'était un peu démesuré ! Mais si tu veux : ce qui c'est passé, c'est qu'on s'est rendu compte que là, on a eu une opposition très forte du bureau protection animale et lutte contre la rage et de l'AFSSA Nancy.» (Un vétérinaire de la hiérarchie de la DGAL)

Mais le $1^{\text {er }}$ septembre $2004^{55}$, le bureau des risques infectieux du ministère de la Santé contacte le bureau de la protection animale à la DGAL pour demander la mise en place de cette vaccination obligatoire. La DGAL répond négativement à cette demande d'extension de la lutte par voie vaccinale. Elle met rapidement en place une proposition alternative qui passe par une extension géographique de la lutte par diverses mesures sanitaires comme l'abattage des animaux errants. Il est proposé en effet d'étendre les mesures applicables dans les sept communes faisant l'objet d'un arrêté préfectoral à l'ensemble des trois départements concernés.

Les mesures sanitaires proposées par la DGAL seront adoptées par un arrêté du ministre de l'Agriculture, le 3 septembre ${ }^{56}$. Cependant le 12 septembre, le ministre de la Santé, P. Douste-Blazy, visite la cellule de crise rage à la préfecture de la Gironde en l'absence du ministre de l'Agriculture qui n'est pas informé de cette initiative. P. Douste-Blazy tient sur place une conférence de presse où il se déclare « favorable à une obligation de vaccination de l'ensemble des carnivores

55. Source : journal de bord de crise à la DGAL.

56. Arrêté du 3 septembre 2004 relatif à des mesures particulières de lutte contre la rage applicables dans les départements de la Dordogne, de la Gironde et du Lot-et-Garonne. 
domestiques sur le territoire national ». Dès le lendemain, la DGAL rédige une note au directeur de cabinet du ministre de l'Agriculture indiquant que la vaccination généralisée serait une réponse coûteuse et inappropriée. Le ministre de l'Agriculture fait sienne la position de ses services et publie un communiqué de presse national sur ce thème. Dans les jours qui suivent, il intervient à de nombreuses reprises sur les ondes nationales et les principales chaînes de télévision française. Alors que la vaccination des carnivores avant le 12 septembre reste un sujet faiblement médiatisé ${ }^{57}$, l'opposition entre les deux ministres sur cette question relance l'activité médiatique et devient le thème majoritaire des articles entre le 12 et le 15 septembre (quarante articles sur les cent vingt-cinq de la période).

Au-delà du débat d'experts sur le caractère approprié ou non de l'obligation vaccinale dans le cadre de l'épisode Tikki, on constate à quel point le nouveau positionnement des autorités de la Santé va influer sur la dynamique de la crise. En effet, l'élargissement des mesures sanitaires qui résulte de la pression exercée par la Santé en matière de vaccination est à l'origine de nombreuses controverses et tensions déjà évoquées (liées notamment à l'interdiction de la chasse sur toute la région Aquitaine et à l'euthanasie des animaux errants) qui vont contribuer à amplifier la crise. Là encore, même si le ministère de l'Agriculture, impose au final sa solution, face au ministère de la Santé, ces différends autour des solutions de gestion de l'alerte contribuent à configurer l'ampleur de cette crise.

\section{Conclusion}

Après cette étude, il paraît évident qu'il n'existe pas de corrélation linéaire entre la gravité épidémiologique du cas Tikki et l'ampleur de la réponse formulée par les autorités et la presse. Même si cet épisode est par nature unique et que des éléments concrets et tangibles viennent conforter l'analyse du risque lié à ce cas, on observe que l'émergence de la crise est fortement liée à des critères organisationnels et cognitifs. En somme, l'histoire de la transformation de l'alerte rage d'août 2004 en crise peut être lue sous l'angle d'une transformation d'un « vieux problème public » en crise du fait de la brusque renégociation du compromis historique tissé autour de la gestion de cette maladie. Les recompositions de la scène de la sécurité sanitaire (via l'apparition de l'InVS et la création du département des urgences sanitaires) vont tout d'abord permettre aux organisations de la Santé de se repositionner au cœur du dispositif de gestion de la rage. Ensuite, la succession des événements sanitaires, la canicule tout d'abord et les différentes alertes rage ensuite, en remodelant tant la perception des crises que leurs enjeux et en permettant l'acquisition de savoirs spécifiques sur la rage au ministère de la Santé, a parallèlement rendu pensable et possible la transformation d'une alerte rage en crise. Enfin, la confrontation de cadres

57. Sept articles essentiellement consacrés à la cohue chez les vétérinaires du Sud-Ouest. 
d'action différents, en matière de recours à la communication et à la vaccination, contribue à reconfigurer le regard et l'analyse du risque sur cette alerte et ainsi à radicaliser les mesures de gestion mises en ouvre. Ainsi, comprendre la crise rage d'août 2004 nécessite de replacer celle-ci à la fois au sein de l'histoire du problème public rage et au cœur des reconfigurations inhérentes à l'ensemble des crises sanitaires. Si les crises sanitaires peuvent être considérées comme des problèmes publics à traiter dans l'urgence, leur compréhension nécessite la prise en compte à la fois de la carrière du problème public propre à chaque crise mais aussi de la catégorie de problèmes publics à laquelle elle se rattache. En ce sens, les crises sanitaires forment une catégorie spécifique de problèmes publics. Les crises peuvent donc difficilement être appréhendées de manière isolée et doivent être réinscrites dans l'histoire même de la catégorie qu'elles contribuent à forger.

Franck Foures est vétérinaire et a suivi l'enseignement du master de sociologie de I'action collective à Sciences-Po Paris. Sa thèse, débutée en octobre 2008 au Centre de sociologie des organisations en collaboration avec I'INRA (Institut national de la recherche agronomique, unité RITME "Risques, Travail, Marché, État »), porte sur les notions d'apprentissage au cours des processus de crise. Les crises sont en effet réputées protéiformes et imprévisibles alors même que les notions de retour d'expérience, de plan d'urgence et de culture du risque, font partie des leitmotivs des organisations confrontées à des alertes successives. Son travail pose ainsi la question suivante : apprend-on vraiment des crises et surtout qu'apprend-on et par quels moyens ? II s'appuie sur l'analyse de crises sanitaires ayant eu plusieurs occurrences soit à des échelons locaux différents (rage 2004 et rage 2008), soit sur un même territoire (méningites à répétition en SeineMaritime). Son travail, en tentant de cerner " l'empreinte " des crises, aide à définir la notion d'expérience des risques et interroge sur l'outil qui donne traditionnellement forme à cette notion, à savoir le " retour d'expérience ".

franck.foures@sciences-po.org 